\title{
Mechanische Partikelabtrennung aus Flüssigkeiten und Gasen
}

\author{
von Dr.-Ing. Harald Anlauf, Institut für Mechanische Verfahrenstechnik \\ und Mechanik, Universität Karlsruhe(TH)
}

Liebe Leserinnen und Leser,

kaum ein Teilgebiet der mechanischen Verfahrenstechnik durchdringt in einer solchen Vielfalt die unterschiedlichsten Bereiche unserer Lebens- und Produktionsumgebung und wird meist dennoch als unspektakuläre Selbstverständlichkeit hingenommen, wie die Abtrennung von Partikeln aus Flüssigkeiten und Gasen. Bei der Reinigung von Abwässern und der Gewinnung von Trinkwasser oder der Luftreinhaltung erfüllt die mechanische Trenntechnik Grundbedürfnisse unserer Existenz und steht immer noch vor großen Herausforderungen. Wer denkt bei biotechnologisch erzeugten Wirkstoffen daran, dass ca. 50-90\% der Herstellkosten durch die Aufbereitungsschritte im sogenannten down stream processing und damit im wesentlichen durch hochspezialisierte und komplexe Trenntechnik bestimmt werden? Nanopartikeln und ihre gezielte Erzeugung und Strukturierung verleihen vielen neuartigen Produkten einzigartige Eigenschaften und sind daher in aller Munde. Hier tritt leicht in den Hintergrund, dass diese Produkte nur dann real zu erzeugen sind, wenn sie in der Prozesskette ihrer Herstellung auch geeignet und wirtschaftlich separiert werden können. Dies ist mit den klassischen Methoden der Trenntechnik oft nur unbefriedigend oder nicht möglich und sowohl die Grundlagenforschung als auch der Apparatebau sind hier gefordert, adäquate Lösungen zu entwickeln. Die Abscheidung nanoskaliger Partikeln erfordert infolge der dominanten Grenzflächenkräfte und der kleinen Strömungsquerschnitte in entsprechenden Partikelschichten eine Modifizierung und Anpassung der bekannten Gesetzmäßigkeiten zur Prozessbeschreibung. Technisch wird auf diese Herausforderungen z. B. mit der Entwicklung immer effizienterer High-Tech-Produkte, Filtermedien aus Nanofasern oder membranbeschichteten Gewebestrukturen reagiert. Partikeldurchmesser von mehreren Zentimetern bis hinab zu wenigen Nanometern, Mehrkomponentengemische, Konzentrationen von wenigen Teilchen im Fluid bis hin zur Fließgrenze, abrasive Mineralteilchen und empfindliche Zellkulturen, in weiten Bereichen variierende physikochemische Eigenschaften von Flüssigkeiten, um Zehnerpotenzen unterschiedliche Viskositäten von Flüssigkeiten und Gasen, Mengenströme von wenigen Millilitern bis hin zu tausenden von Kubikmetern pro Stunde, sowie unterschiedlichste Anforderungen an das Trennergebnis führen immer wieder $\mathrm{zu}$ weder in der Theorie noch in der technischen Praxis gelösten Trennproblemen.

Zur Meisterung derartiger Herausforderungen ist heute in vielen Fällen eine interdisziplinäre Zusammenarbeit von Naturwissenschaftlern und Ingenieuren unabdingbar. Weiterhin stehen mit den sich rasant entwicklenden und heute kaum noch wegzudenkenden rechnerischen Simulationsmethoden wichtige Werkzeuge zur Problemlösung und -optimierung zur Verfügung. Diese werden auch bereits auf breiter Front im Bereich der mechanischen Trenntechnik eingesetzt. Die Simulation wird jedoch niemals den kreativen Verstand des Ingenieurs ersetzen können, der die „zündende Idee“ zur neuartigen oder verbesserten Lösung einer Trennaufgabe hervorbringt.

Die erfreulich große Resonanz auf die Idee, mechanische Trenntechnik in einer CIT-Ausgabe zum Schwerpunkt zu machen, spiegelt sich in den vielfältigen Beiträgen dieses Heftes wider, welche die oben genannten Aspekte illustrieren und ich möchte den Autoren von dieser Stelle aus sehr für ihre Mitarbeit danken.

Dieses Schwerpunktheft belegt, wie breit und wie intensiv und auf wie vielen Gebieten an diesen Themen in For-

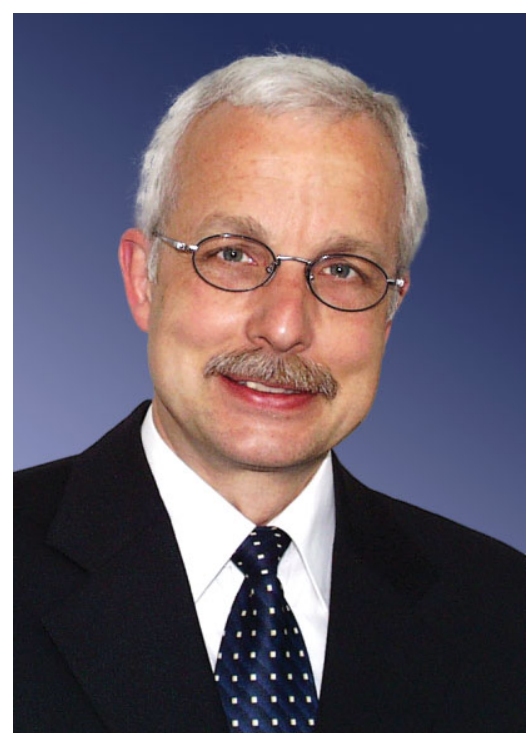

schung und Entwicklung gearbeitet wird. Im April 2008 wird sich die weltweite Fachgemeinschaft der Trenntechniker in Leipzig zum 10. World Filtration Congress versammeln. Deutschland richtet diesen Kongress mit begleitender Fachausstellung zum ersten Mal aus. Mit allein über 350 Fachbeiträgen auf dem Kongress bietet sich die einmalige Gelegenheit, konzentriert einen kompletten weltweiten Überblick über den Stand von Wissenschaft und Technik der mechanischen Partikeltrennprozesse $\mathrm{zu}$ gewinnen und neueste Ergebnisse und Entwicklungen zu diskutieren.

Ich würde mir wünschen, dass Sie als Leserinnen und Leser durch dieses Schwerpunktheft fachlich gut informiert und angeregt werden und ich würde mich freuen, Sie nach dieser Vorbereitung 2008 in Leipzig auf dem WFC10 zur weiteren Vertiefung des Themas begrüßen zu dürfen.

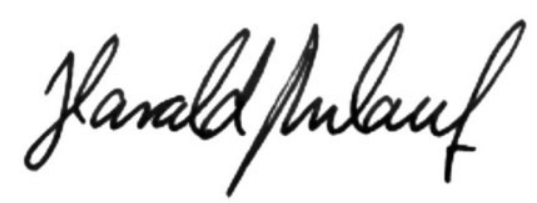

